\title{
Comparison of Macrophage Activation Using $\gamma$-Globulin and Serum-derived Macrophage Activating Factor
}

\author{
MINORI AKAMATSU, RIHO MASHIBA, KUMPEI KAWAKATSU, MAMI ISHIKAWA ${ }^{\#}$ and TAKAHITO NISHIKATA
}

Frontiers of Innovative Research in Science and Technology (FIRST), Konan University, Kobe, Japan

\begin{abstract}
Background/Aim: Serum-derived macrophage activating factor (serum-MAF) can rapidly activate macrophage phagocytic activity by inducing characteristic membrane ruffles designated as Frill-like structures. SerumMAF contains $\gamma$-globulin, an activator of phagocytosis. This study examined whether serum-MAF and $\gamma$-globulin activate macrophages similarly. Materials and Methods: Morphological changes in macrophages were observed by time-lapse imaging and the efficiency of engulfment was analysed quantitatively. Immunological staining of talin-1 and a calpain inhibitor were performed. Results: The engulfment efficiency of serum-MAFand $\gamma$-globulin-activated macrophages was significantly different. Talin-1 showed weak co-localisation with the Frilllike structures. Treatment with a calpain inhibitor similarly down-regulated phagocytosis irrespective of the activation factor. Conclusion: There was a difference between macrophage activation mechanisms by $\gamma$-globulin and serumMAF. Talin may slightly contribute to serum-MAF activation. It is possible to distinguish between the calpain-dependent fundamental 'mechanism of phagocytosis' and the activating factor-dependent rapid 'activation mechanism'.
\end{abstract}

In the innate immune system, macrophages recognise and phagocytose pathogens, present antigen information, and release cytokines to initiate appropriate immune responses (1). For initiating such self-defence mechanisms, a rapid reaction against the invading agent is important. In our

This article is freely accessible online.

\#Present address: Aeras Bio Inc., Kobe, Japan.

Correspondence to: Takahito Nishikata, Frontiers of Innovative Research in Science and Technology (FIRST), Konan University, 7-1-20, Minatojima-minamimachi, Chuo-ku, Kobe 650-0047, Japan. Tel: +81 783031349, Fax: +81 783031495, e-mail: nisikata@konanu.ac.jp

Key Words: THP-1 macrophages, serum-MAF, $\gamma$-globulin, talin-1, calpain, time-lapse imaging, morphological change. previous study, serum-derived macrophage activating factor (serum-MAF), which is human serum treated with $\beta$ galactosidase and sialidase, evoked a rapid and strong activation of phagocytosis with the occurrence of unique and prominent membrane ruffles designated as Frill-like structures (2). Frill-like structures are multi-layered membrane ruffles characterized by $\mathrm{F}$-actin accumulation. Although lipopolysaccharides with interferon- $\gamma$, common macrophage activators, can activate phagocytosis, their phagocytic activation is significantly less than that of serumMAF, suggesting different activation mechanisms (3).

Two major phagocytic mechanisms (Fc $\gamma$ Rs-mediated and $\alpha \mathrm{M} \beta 2$-integrin-mediated) have been well-documented (4). As serum-MAF is made from the human serum, it contains a substantial amount of $\mathrm{IgG}$ (approximately $10 \mathrm{mg} / \mathrm{ml}$ ). Thus, it is important to distinguish between an IgG-mediated and a serum-MAF-mediated activation. First, in this study, using time-lapse recordings, the characteristic phagocytic activation behaviours of macrophages by $\gamma$-globulin and serum-MAF were compared. Subsequently, because one of the candidate mechanisms for mediating serum-MAF activation is the 'inside-out' signal of integrin and talin (5, $6)$, and talin is a substrate of calpain [a calcium-dependent cysteine protease (7)], we focussed on talin-1 localisation and the effect of calpain.

\section{Materials and Methods}

Cell culture. Culture and differentiation conditions of THP-1 cells (RIKEN BRC, Tokyo, Japan) were performed as a previously described (2). Differentiated macrophages were activated with 2 $\mu \mathrm{g} / \mathrm{ml}$ human $\gamma$-globulin (Sigma-Aldrich, St. Louis, MO, USA) or $6 \mu \mathrm{g} / \mathrm{ml}$ serum-MAF (Saisei-mirai Clinic, Kobe, Japan) for $1 \mathrm{~h}$. After washing with serum-free RPMI-1640 medium, macrophages were incubated in $3 \mu \mathrm{g} / \mathrm{ml}$ magnetic beads (Dynabeads Protein G; Invitrogen, Waltham, MA, USA) containing serum-free RPMI1640 for $1 \mathrm{~h}$ and evaluated quantitatively for their phagocytic activities (8).

THP-1 cells expressing the LifeAct-green fluorescent protein (LifeAct-THP-1). LifeAct-THP-1 cells that stably express green fluorescent protein were obtained by retroviral infection (9). 


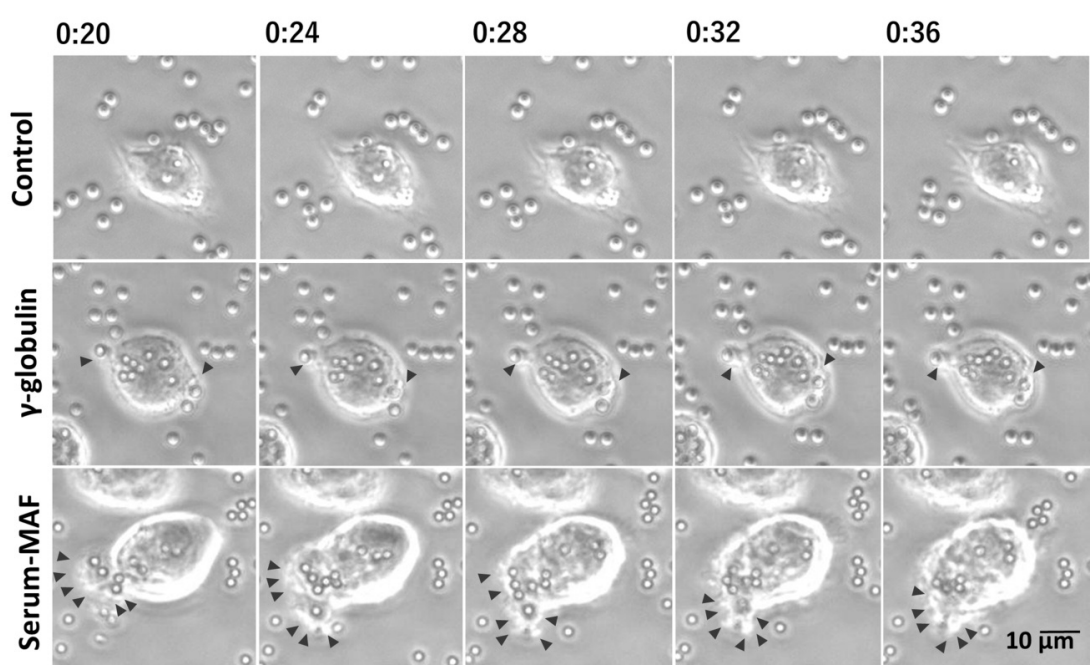

Figure 1. Time-lapse imaging of bead engulfment by macrophages. Morphological changes of macrophages treated with or without the activation factors were compared from 20 to 36 min after addition of the beads (the numbers in each panel indicate min). The small particles around the cell body are magnetic beads. Arrowheads indicate extended membrane ruffles around the beads that were being actively engulfed, suggesting that these are phagocytic cups and Frill-like structures. Scale $=10 \mu \mathrm{m}$.

Time-lapse imaging and efficiency of engulfment (EE). THP-1 cells were seeded at $5.0 \times 10^{5}$ cells in a $3.5-\mathrm{cm}$ dish, then differentiated, activated, and incubated with beads as described in the Cell culture section. Immediately after the addition of the beads, time-lapse recordings of three fields within each dish were performed every 4 min for $1 \mathrm{~h}$ by a phase contrast microscopy (BZ-9000 BioRevo; Keyence, Osaka, Japan). The numbers of contacted (C\#) and phagocytosed ( $\mathrm{P \# )}$ beads were counted manually in all frames. The EE was calculated using equation 1 :

\section{$E E(\%)=(P \# / C \#) \times 100$}

Confocal microscopy observations. Cells were seeded on small cover slips $(24 \times 6 \mathrm{~mm})$ within a $3.5-\mathrm{cm}$ dish and processed as described in the Cell culture section. After activation for $1 \mathrm{~h}$, cells were fixed with $4 \%$ paraformaldehyde in phosphate-buffered saline (PBS) for $10 \mathrm{~min}$ at $20-25^{\circ} \mathrm{C}$. Next, cells were permeabilised with $0.2 \%$ Triton X-100 in PBS for 5 min. After blocking with 5\% bovine serum albumin (Sigma-Aldrich; A3059) for $1 \mathrm{~h}$, cells were immunostained with an anti-talin-1 antibody (Proteintech, Rosemont, IL, USA; 14168-1-AP; 1:100), Alexa Fluor plus 555 anti-mouse IgG (Invitrogen; A32732; 1:1,000), Alexa Fluor ${ }^{\mathrm{TM}} 488$ phalloidin (Invitrogen; A12379; $6.6 \mathrm{U} / \mathrm{ml}$ ), and $10 \mu \mathrm{g} / \mathrm{ml} \mathrm{4',6-}$ diamidino-2-phenylindole. Cells were observed under an A1R HD25 confocal microscope (Nikon, Tokyo, Japan).

Assays for phagocytic activity and calpain inhibition. THP-1 cells were seeded in 96 -well plates at a density of $2.0 \times 10^{4}$ cells per well. Differentiated macrophages were activated with or without $100 \mathrm{nM}$ MDL-28170 (a calpain-1 and -2 inhibitor; Cayman Chemical, Ann Arbor, MI, USA; 14921) for $1 \mathrm{~h}$, and their phagocytic activities were evaluated as reported by Ishikawa et al. (8).

Statistical analysis. All values are presented as mean \pm SD. Statistical analysis was performed using one-way ANOVA and Fisher's least

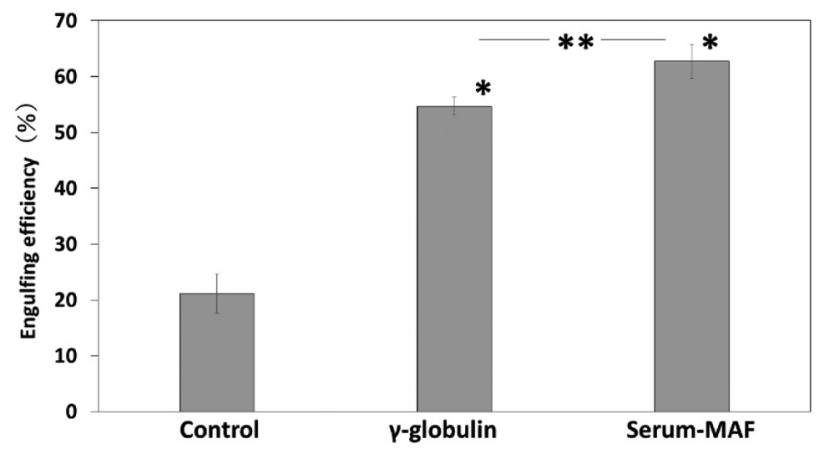

Figure 2. Engulfment efficiency (EE) of the macrophages. The EE was estimated from time-lapse movies of the movement of macrophages activated by $\gamma$-globulin, serum-derived macrophage activating factor (serum-MAF), or without activation factor (control). ${ }^{*} p<0.01$ compared to control and ${ }^{* *} p<0.05$ comparing $\gamma$-globulin to serum-MAF.

significant difference test. $p$-Values of $<0.05$ were considered to be statistically significant.

\section{Results}

Different phagocytic activities of serum-MAF- and $\gamma$-globulinactivated macrophages. To compare the phagocytic behaviours of un-activated or activated macrophages, time-lapse images were analysed (Figure 1). When macrophages were activated by $\gamma$-globulin, small membrane extrusions were observed beneath the attached beads. Serum-MAF-activated macrophages showed active membrane ruffling, like that in the Frill-like structures reported by Kawakatsu et al. (2019). At the 


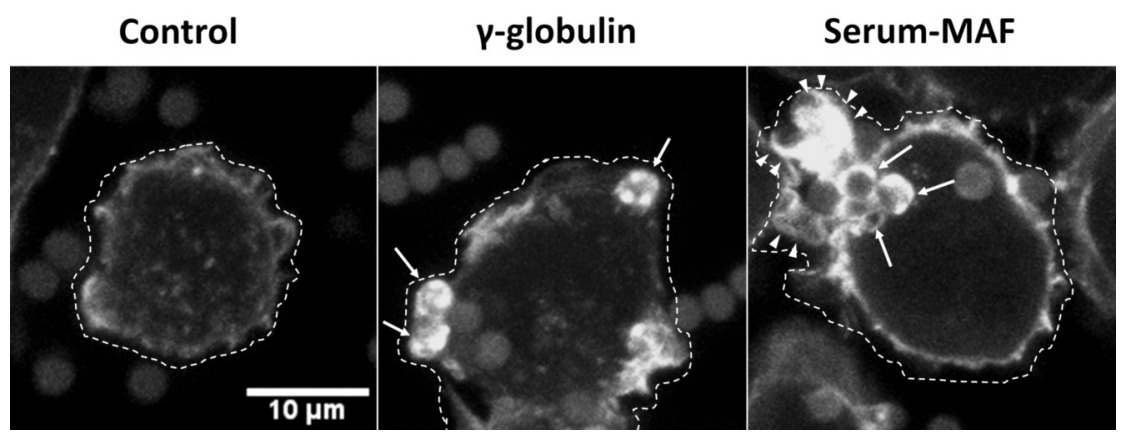

Figure 3. F-Actin accumulation in membrane ruffles. The LifeAct-green fluorescent protein (LifeAct)-THP-1 macrophages were activated with or without the activation factors and incubated with beads. Images of single confocal microscopic optical sections at 2.4 um from the substrate surface are shown. Thin broken lines indicate cell borders. Arrows indicate beads that were engulfed and surrounded by F-actin of the phagocytic vesicle. Arrowheads indicate the extended and F-actin-accumulated lamellipodia. Scale $=10 \mu \mathrm{m}$.

Control
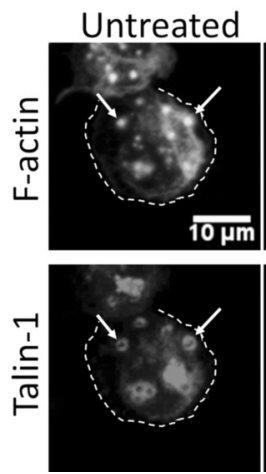

Y-globulin
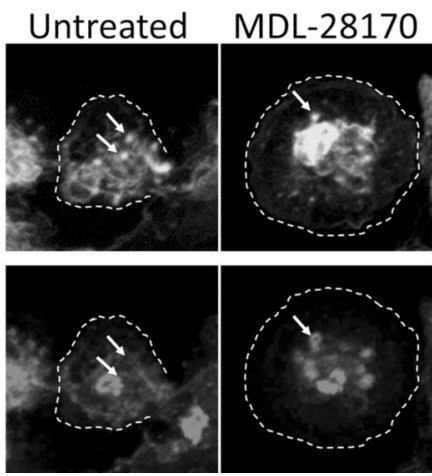

Serum-MAF

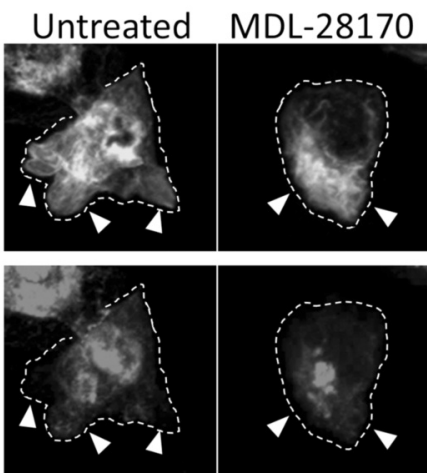

Figure 4. Effect of a calpain inhibitor on F-actin accumulation and talin localisation. THP-1 macrophages were incubated with or without the activation factors and a calpain inhibitor (MDL-28170) without the addition of magnetic beads. Fixed cells were immunostained with an anti-talin antibody and stained with phalloidin. Thin broken lines indicate cell borders. Arrows and arrowheads indicate podosomes and Frill-like structures, respectively. Scale $=10 \mu \mathrm{m}$.

site of this membrane ruffling, several beads were actively engulfed. Moreover, the EE of the serum-MAF-activated macrophages was significantly greater than those of both unactivated and $\gamma$-globulin-activated macrophages (Figure 2; $p<0.001$ and $p=0.029$, respectively).

Figure 3 shows that in the $\gamma$-globulin-activated LifeActTHP-1 macrophages, newly engulfed beads were covered with F-actin, suggesting the formation of phagocytic cups and vesicles. Serum-MAF-activated macrophages showed strong accumulation of F-actin on the extended membrane ruffles corresponding to the Frill-like structure. Beneath the F-actin accumulation, the F-actin surrounded several beads, suggesting an active engulfment.

Relationship between phagocytic cell activation and talin-1 colocalisation. Co-localisation of talin-1 and actin was examined (Figure 4). In the control and $\gamma$-globulin-activated macrophages, talin-1 was found to be localised around the podosome.

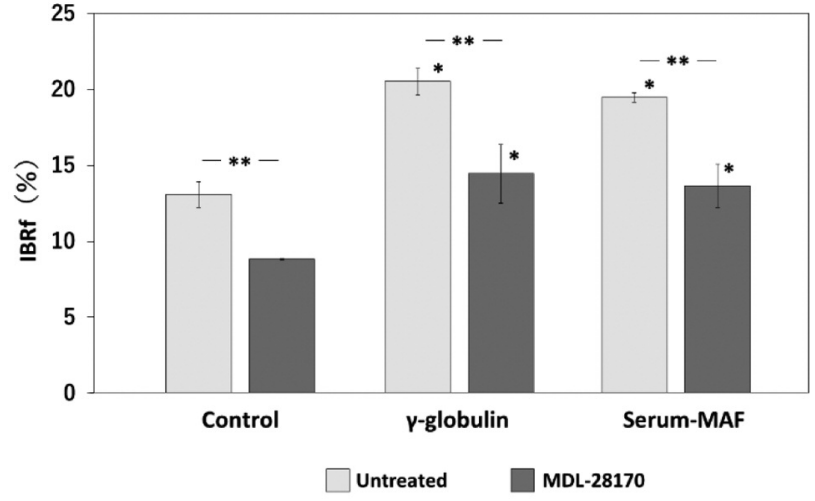

Figure 5. Effect of a calpain inhibitor on phagocytic activity. THP-1 macrophages were incubated with or without the activation factors and a calpain inhibitor (MDL-28170). Phagocytic activities were measured by fluorescence (IBRf). * $p<0.01$ compared to control and **p<0.01 comparisons with and without the calpain inhibitor. 
However, in serum-MAF-activated macrophages, podosomes disappeared and Frill-like structures were formed. In these cells, talin-1 showed a weak co-localisation within some Frill-like structures. When the calpain inhibitor, MDL-28170, was added to these macrophages, co-localisation of actin and talin- 1 became weak, although the overall cell morphology and localisation of actin and talin in each activation condition did not change. Phagocytic activity under calpain-inhibition conditions (addition of MDL-28170) of un-activated, $\gamma$-globulin-activated, and serum-MAF-activated macrophages was down-regulated evenly (Figure 5: $32.6 \%, 29.6 \%$, and 29.9\%, respectively) compared to the activity under conditions without the inhibitor.

\section{Discussion}

Actin, integrin, talin, and calpain are key components for the phagocytosis of macrophages (10). In THP-1 macrophages, talin-1 and calpain-2 are the major isoforms (11). Thus, our calpain inhibition results suggest that talin-1 and calpain-2 are mediators of THP-1 phagocytic activity. However, as the target spectrum of calpains is wide, this result did not directly demonstrate a role for talins. Likewise, Kumar et al. (12) reported that peritoneal macrophages from calpain knockout mice could phagocytose bacteria, but showed an impaired clearance of intracellular bacteria.

In this study, time-lapse analyses revealed that the rapid phagocytic activation mechanisms underlying $\gamma$-globulin- and serum-MAF-activated macrophages differed. Furthermore, the Frill-like structures were responsible for the high EE achieved by serum-MAF. While a calpain-related mechanism can be implicated in various types of macrophage phagocytosis, these results clearly suggest that the mechanisms of 'activation' and 'phagocytosis' should be considered separately. This concept may be helpful for elucidating the phagocytic activation mechanism.

\section{Conflicts of Interest}

The Authors have declared that no conflicts of interest exist in relation to this study.

\section{Authors' Contributions}

TN conceived and designed the study, and assisted in manuscript preparation. MA performed the experiments and wrote the manuscript draft. All Authors contributed to data analysis and interpretation, and critically reviewed the manuscript. The final version of the manuscript was approved by all Authors.

\section{Acknowledgements}

The Authors would like to thank Dr. T. Inui (Inui Immunotherapy Clinic, Osaka, Japan) and Dr. Y. Uto (Tokushima University Graduate School, Tokushima, Japan) for providing the serum-MAF. The Authors are also grateful to Dr. T. Iwasaki (Kobe University,
Kobe, Japan) and Dr. T. Taniguchi (Pharma Crea Kobe Co.Ltd., Kobe, Japan) for their continuous discussion. This work was partly supported by the joint research program of Biosignal Research Center, Kobe University and Pharma Crea Kobe Co.Ltd.

\section{References}

1 Medzhitov R and Janeway C: Innate immunity. N Engl J Med 343(5): 338-344, 2000. PMID: 10922424. DOI: 10.1056/ NEJM200008033430506

2 Kawakatsu K, Ishikawa M, Mashiba R, Tran NK, Akamatsu M and Nishikata T: Characteristic morphological changes and rapid actin accumulation in serum-MAF-treated macrophages. Anticancer Res 39(8): 4533-4537, 2019. PMID: 31366556. DOI: 10.21873/anticanres. 13630

3 Mashiba R, Ishikawa M, Sumiya Y, Kawakatsu K, Tran NK and Nishikata T: Phagocytic activation of macrophages with serum MAF depends on engulfment efficiency and not migratory activity. Anticancer Res 38(7): 4295-4298, 2018. PMID: 29970564. DOI: 10.21873 /anticanres.12727

4 Rougerie P, Miskolci V and Cox D: Generation of membrane structures during phagocytosis and chemotaxis of macrophages: role and regulation of the actin cytoskeleton. Immunol Rev 256(1): 10.1111/imr.12118, 2013. PMID: 24117824. DOI: 10.1111/imr.12118

5 Lim J, Wiedemann A, Tzircotis G, Monkley SJ, Critchley DR and Caron E: An essential role for talin during alphaMbeta2mediated phagocytosis. Mol Biol Cell 18: 976-985, 2007. PMID: 18363565. DOI: 10.1091/mbc.E06-09-0813

6 Medraño-Fernandez I, Reyes R, Olazabal I, Rodriguez E, SanchezMadrid F, Boussiotis VA, Reche PA, Cabañas C and Lafuente EM: RIAM (Rap1-interacting adaptor molecule) regulates complementdependent phagocytosis. Cell Mol Life Sci 70(13): 2395-2410, 2013. PMID: 23420480. DOI: 10.1007/s00018-013-1268-6

7 Jeong SY, Martchenko M and Cohen SN: Calpain-dependent cytoskeletal rearrangement exploited for anthrax toxin endocytosis. Proc Natl Acad Sci USA 110(42): E4007-4015, 2013. PMID: 24085852. DOI: 10.1073/pnas.1316852110

8 Ishikawa M, Mashiba R, Kawakatsu K, Tran NK and Nishikata T: A high-throughput quantitative assay system for macrophage phagocytic activity. Macrophage 5: e1627, 2018. DOI: 10.14800/macrophage. 1627

9 Kawauchi K, Araki K, Tobiume K and Tanaka N: p53 regulates glucose metabolism through an IKK-NF-kB pathway and inhibits cell transformation. Nat Cell Biol 10(5): 611-618, 2008. PMID: 18391940. DOI: 10.1038/ncb1724

10 Patel PC and Harrison RE: Membrane ruffles capture C3biopsonized particles in activated macrophages. Mol Biol Cell 19: 4628-4639, 2008. PMID: 18768756. DOI: 10.1091/mbc.e08-020223

11 Sumiya Y, Ishikawa M, Inoue T, Inui T, Kuchiike D, Kubo K, Uto $\mathrm{Y}$ and Nishikata T: Macrophage activation mechanisms in human monocytic cell line-derived macrophages. Anticancer Res 35: 4447-4451, 2015. PMID: 26168485.

12 Kumar V, Everingham S, Hall C, Greer PA and Craig AW: Calpains promote neutrophil recruitment and bacterial clearance in an acute bacterial peritonitis model. Eur J Immunol 44(3): 831-841, 2014. PMID: 24375267. DOI: 10.1002/eji.201343757

Received May 23, 2020

Revised June 18, 2020

Accepted June 20, 2020 